\title{
Developing a clinical development paradigm for translation of a mammaglobin-A DNA vaccine
}

\begin{abstract}
"...we are convinced that analysis of the immune response in the primary tumor following vaccination is likely to be significantly more informative than studies of the immune response in the peripheral blood."
\end{abstract}

Keywords: breast cancer $\bullet$ clinical trial $\bullet$ DNA vaccine $\bullet$ immunotherapy $\bullet$ mammaglobin-A cer vaccine therapy [7]. Cancer vaccines are typically designed to stimulate the immune system to mount a cell-mediated immune response against one or more tumor-associated antigens. Cancer vaccines have excellent safety profiles and several have now received US FDA approval including Gardasil and Cervarix for prevention of cervical cancer and Provenge for treatment of metastatic prostate cancer. Investigators are now pursuing several different cancer vaccine platforms including autologous cellular vaccines, DNA vaccines and protein or peptide vaccines, often in combination with conventional cancer treatment regimens. We recently completed an open-label Phase I clinical trial (Clinicaltrials.gov identifier: NCT00807781) evaluating the safety and immunogenicity of a DNA vaccine designed to express the human breast cancer-associated mammaglobin-A antigen. Results of the trial demonstrate the safety of the mammaglobin-A DNA vaccine, with preliminary evidence to suggest that the mammaglobin-A vaccine is able to induce mammaglobin-A-specific IFN- $\gamma$-secreting CD8 T cells in treated patients. These patients also had improved progression-free survival [8].

\section{Mammaglobin-A is an exceptional target for breast cancer vaccine therapy}

Mammaglobin-A is a member of the secretoglobin superfamily (family $2 \mathrm{~A}$ member 2). The mammaglobin-A gene (SCGB2A2) was first identified using a differential screen-

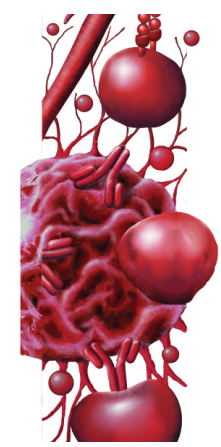

Lijin Li

Department of Surgery, Washington University School of Medicine, St Louis, MO 63110, USA

\section{S Peter Goedegebuure}

Department of Surgery, Washington University School of Medicine, St Louis, MO 63110, USA, and,

The Alvin J Siteman Cancer Center at Barnes-Jewish Hospital \& Washington University School of Medicine, MO, USA

Timothy P Fleming

Department of Surgery, Washington University School of Medicine, St Louis, MO 63110, USA, and,

The Alvin J Siteman Cancer Center at Barnes-Jewish Hospital \& Washington University School of Medicine, MO, USA

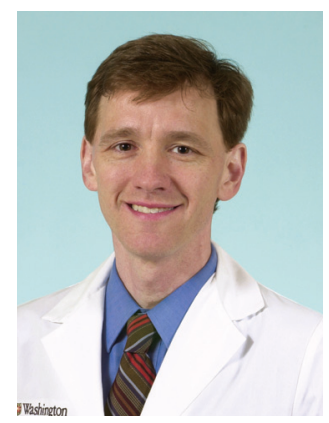

William E Gillanders Author for correspondence: Department of Surgery, Washington University School of Medicine, St Louis, MO 63110, USA, and,

The Alvin J Siteman Cancer Center at Barnes-Jewish Hospital \& Washington University School of Medicine, MO, USA Tel.: +1 3147470072 Fax: +1 3144545509 gillandersw@wustl.edu

Future $\because$ Medicine part of 
ing approach directed at the isolation of novel human breast cancer-associated genes [9]. Since its original identification, mammaglobin-A has proven to be an important molecular marker for the detection of metastatic breast cancer, and it has been the focus of over 300 publications in the medical literature. The exquisite tissue specificity and near universal overexpression of mammaglobin-A makes it an attractive target for a breast cancer vaccine with potential for use in a breast cancer prevention and/or treatment setting. Evidence of preexisting immunity to mammaglobin-A in breast cancer patients, and strong preclinical data provided additional rationale for clinical translation of a DNA vaccine targeting mammaglobin-A.

\section{Phase I clinical trial of a mammaglobin-A DNA vaccine demonstrates the safety of the vaccine, with strong preliminary evidence of biologic efficacy}

A total of 53 patients met all of the eligibility criteria and were enrolled in a Phase I clinical trial between December 2009 and May 2013 [8]. After enrollment, 39 patients were considered screen failures and 14 patients were vaccinated. The mammaglobin-A DNA vaccine was remarkably safe, and no significant adverse events were observed. Eight of the 14 vaccinated patients were HLA-A2 ${ }^{+}$and were studied with in-depth immune monitoring analyses using peripheral blood mononuclear cells collected before, during and after vaccination. Following vaccination there was a significant increase in the frequency of CD8/MAMA2.1 $1^{+} \mathrm{T}$ cells as measured by flow cytometry $(0.9 \pm$ $0.5 \%$ vs $3.8 \pm 1.2 \% ; \mathrm{p}<0.001)$. ELISPOT analysis similarly demonstrated a significant increase in the number of mammaglobin-A specific IFN- $\gamma$ producing CD8 T cells ( $41 \pm 32$ vs $215 \pm 67$ spm; $\mathrm{p}<0.001)$. A comparison between vaccinated patients and patients who were screen failures based on HLA type suggested that mammaglobin-A DNA vaccination was associated with improved progression-free survival [8].

\section{Future perspective}

Recent experience has provided significant insights into the challenges associated with clinical translation of cancer vaccines. Initial cancer vaccine studies were typically performed in the context of metastatic cancer, using response criteria that were developed for cytotoxic therapies. In addition, surrogates for vaccine efficacy remain imperfect. We have tried to address these limitations in our ongoing studies of the mammaglobin-A DNA vaccine.

First, cancer vaccines are very different from cytotoxic chemotherapies, and the clinical development paradigm for cancer vaccines is very different. The clinical development paradigm for cytotoxic agents involves initial testing in patients with metastatic disease to determine the maximum tolerated dose (Phase I), and then to assess biologic efficacy (Phase II). However, it is now commonly believed that generation of an effective antitumor immune response will be difficult in patients with metastatic cancer [10]. Metastatic breast cancer is no exception; metastatic breast cancer is associated with an increase in the prevalence of regulatory $\mathrm{T}$ cells $[11,12]$ and immature myeloid cells $[13,14]$ in the peripheral blood and in the tumor microenvironment, and these cells are capable of inhibiting endogenous or elicited antitumor immune responses. Patients with metastatic breast cancer may also have compromised immune systems related to prior cytotoxic therapy. The current clinical development paradigm in the cancer vaccine field emphasizes the early assessment of vaccine efficacy in an appropriate clinical context [15], and this paradigm has been endorsed by the FDA. As detailed below, vaccination in the context of neoadjuvant endocrine therapy is an appropriate clinical context to assess breast cancer vaccine efficacy.

Second, current surrogates for vaccine efficacy are imperfect. Many cancer vaccines have successfully induced immune responses in the peripheral blood, but have failed to induce an antitumor response. It is now known that regulatory networks present in the tumor microenvironment restrain antitumor immune responses. One of the limitations of almost all current cancer vaccine trials is that assessment of biologic efficacy is dependent on imperfect surrogates (immune response in the peripheral blood), and a detailed analysis of antitumor immune responses in the primary tumor is not possible. We believe that there is an excellent opportunity to test the efficacy of breast cancer vaccines in the setting of neoadjuvant endocrine therapy. Breast cancer patients with clinical T2 N0 estrogen receptor-positive breast cancer are often treated with neoadjuvant endocrine therapy (aromatase inhibitors) with the goal of decreasing tumor size and facilitating breast conservation therapy. This provides an excellent window of opportunity to treat patients with an investigational breast cancer vaccine and then rigorously assess antitumor immune responses both in the peripheral blood and in the primary tumor.

Finally, the DNA vaccine platform is a remarkably flexible platform as DNA vaccines are relatively easy to manufacture, with the ability to genetically manipulate the encoded antigens, and/or incorporate other genes to amplify the immune response. Of note, the recent development of electroporation devices that can be used in human clinical trials has increased the potential for clinical translation of DNA vaccines, as electroporation can dramatically increase DNA uptake 
by muscle cells, antigen expression and immunogenicity [16-19]. The importance of electroporation to the successful clinical translation of DNA vaccines was recently highlighted and emphasized in a high-profile review [20], and in the introduction to a special issue of Vaccine [21].

Building on the success of our Phase I clinical trial, we have recently initiated a Phase Ib clinical trial of the mammaglobin-A DNA vaccine in patients receiving neoadjuvant endocrine therapy. We will use immunohistochemistry, flow cytometry and molecular analyses to study the impact of mammaglobin-A DNA vaccination on the primary tumor. To date, very few cancer vaccine trials have been performed in the neoadjuvant setting, and we are convinced that analysis of the immune response in the primary tumor following vaccination is likely to be significantly more informa-

\section{References}

1 American Cancer Society. Cancer Facts \& Figures 2015. Atlanta: American Cancer Society (2015).

2 Vesely MD, Schreiber RD. Cancer immunoediting: antigens, mechanisms, and implications to cancer immunotherapy. Ann. NYAcad. Sci. 1284, 1-5 (2013).

3 Hanahan D, Weinberg RA. Hallmarks of cancer: the next generation. Cell 144(5), 646-674 (2011).

4 Couzin-Frankel J. Breakthrough of the year 2013. Cancer immunotherapy. Science 342(6165), 1432-1433 (2013).

5 Maude SL, Frey N, Shaw PA et al. Chimeric antigen receptor T cells for sustained remissions in leukemia. N. Engl. J. Med. 371(16), 1507-1517 (2014).

6 Wolchok JD, Kluger H, Callahan MK et al. Nivolumab plus Ipilimumab in advanced melanoma. N. Engl. J. Med. 369(2), 122-133 (2013).

7 Senovilla L, Vacchelli E, Garcia P et al. Trial watch: DNA vaccines for cancer therapy. Oncoimmunology 2(4), e23803 (2013).

8 Tiriveedhi V, Tucker N, Herndon J et al. Safety and preliminary evidence of biologic efficacy of a mammaglobin-a DNA vaccine in patients with stable metastatic breast cancer. Clin. Cancer Res. 20(23), 59645975 (2014).

9 Watson MA, Fleming TP. Isolation of differentially expressed sequence tags from human breast cancer. Cancer Res. 54(17), 4598-4602 (1994).

10 Finn OJ. Cancer vaccines: between the idea and the reality. Nat. Rev. Immunol. 3(8), 630-641 (2003).

11 Liyanage UK, Moore TT, Joo HG et al. Prevalence of regulatory $\mathrm{T}$ cells is increased in peripheral blood and tumor microenvironment of patients with pancreas or breast adenocarcinoma. J. Immunol. 169(5), 2756 (2002). tive than studies of the immune response in the peripheral blood, providing important insights into how to improve current cancer vaccine platforms.

\section{Financial \& competing interests disclosure}

WE Gillanders was supported by research grants DOD/CDMRP-BCRP W81XWH-06-1-0677 and Gateway for Cancer Research P-06-016. SP Goedegebuure was supported by the Foundation for Barnes-Jewish Hospital. TP Fleming holds a patent on mammaglobin-A that is owned by Washington University. The authors have no other relevant affiliations or financial involvement with any organization or entity with a financial interest in or financial conflict with the subject matter or materials discussed in the manuscript apart from those disclosed.

No writing assistance was utilized in the production of this manuscript.

12 Wolf AM, Wolf D, Steurer M, Gastl G, Gunsilius E, Grubeck-Loebenstein B. Increase of regulatory T cells in the peripheral blood of cancer patients. Clin. Cancer Res. 9(2), 606-612 (2003).

13 Almand B, Clark JI, Nikitina E et al. Increased production of immature myeloid cells in cancer patients: a mechanism of immunosuppression in cancer. J. Immunol. 166(1), 678-689 (2001).

14 Almand B, Resser JR, Lindman B et al. Clinical significance of defective dendritic cell differentiation in cancer. Clin. Cancer Res. 6(5), 1755-1766 (2000).

15 Hoos A, Parmiani G, Hege K et al. A clinical development paradigm for cancer vaccines and related biologics. J. Immunother. (1997) 30(1), 1-15 (2007).

16 Aihara H, Miyazaki J. Gene transfer into muscle by electroporation in vivo. Nat Biotechnol 16(9), 867-870 (1998).

17 Mathiesen I. Electropermeabilization of skeletal muscle enhances gene transfer in vivo. Gene Ther. 6(4), 508-514 (1999).

18 Mir LM, Bureau MF, Gehl J et al. High-efficiency gene transfer into skeletal muscle mediated by electric pulses. Proc. Natl Acad. Sci. USA 96(8), 4262-4267 (1999).

19 Widera G, Austin M, Rabussay D et al. Increased DNA vaccine delivery and immunogenicity by electroporation in vivo. J. Immunol. 164(9), 4635-4640 (2000).

20 Rice J, Ottensmeier CH, Stevenson FK. DNA vaccines: precision tools for activating effective immunity against cancer. Nat. Rev. Cancer 8(2), 108-120 (2008).

21 Weiner DB. DNA vaccines: crossing a line in the sand introduction to special issue. Vaccine26(40), 5073-5074 (2008). 